\title{
MORTALIDADE POR CÂNCER DE PRÓSTATA NA REGIÃO NORDESTE DO BRASIL: UM OLHAR SOBRE A SAÚDE DO HOMEM
}

\author{
PROSTATE CANCER MORTALITY IN THE NORTHEAST REGION OF \\ BRAZIL: A LOOK INTO MEN'S HEALTH.
}

\author{
Andreza Silva Costa ${ }^{1}$ \\ Tais Layane de Sousa Lima² \\ Bruna Braga Dantas ${ }^{3}$
}

RESUMO: OBJETIVO: Avaliar o perfil de comportamento da taxa de mortalidade (TM) por câncer de próstata (CP) na região Nordeste, considerando o período de 1988 à 2017, assim como a progressão da TM para o período de 2018 à 2067. METODOLOGIA: Para isto, foi realizado um estudo documental e descritivo, baseado em dados coletados no Atlas de Mortalidade sobre a TM por câncer de próstata da população nordestina no período de 1988 à 2017, e a partir destes dados foi calculado a progressão da mortalidade por esta neoplasia para o período 2018 à 2067. RESULTADOS: Durante o período analisado (1988 à 2017) observouse que a mortalidade por CP teve aumentos contínuos, tornando o Nordeste a segunda região do Brasil com maior TM por CP na última década de estudos (2008 à 2017), e ao estimar a progressão da mortalidade por CP no período de 50 anos constatou-se que a mesma tende a aumentar de 33,17 para 689,28 ,no período de 2018 á 2067, respectivamente, sendo ela maior que a apresentada na progressão da TM por câncer de mama no período de 90 anos na referida região. CONCLUSÃO: Desse modo conclui-se que se faz necessário implementar medidas e ações referentes à saúde do homem, visando regredir a TM por CP. Tornando-se visível que, caso contrário, essa taxa tende a se elevar amplamente nos anos subsequentes.

\footnotetext{
1 Graduanda em Enfermagem pela Universidade Federal de Campina Grande - UFCG/CES.

2 Graduanda em Enfermagem pela Universidade Federal de Campina Grande - UFCG/CES.

${ }^{3}$ Bacharelado em Enfermagem pela Universidade Federal da Paraíba (2011), especialista em Saúde Coletiva pela Faculdade Integrada de Patos (2011), mestre em Farmacologia de Produtos Naturais e Sintéticos Bioativos (2014) e doutorado em Biotecnologia - RENORBIO (2018), ambos pela Universidade Federal da Paraíba. Atualmente, é Professora Adjunta da Unidade Acadêmica de Saúde da Universidade Federal de Campina Grande - Campus Cuité e Professora Assistente da Faculdade Nova Esperança (FACENE). Tem experiência em pesquisa na área de farmacologia celular e molecular, com ênfase na avaliação da atividade citotóxica e anticâncer de moléculas naturais e sintéticas.
} 
Palavras chave: Câncer de Próstata; Prevenção; Saúde do homem.

ABSTRACT: OBJECTIVE: To evaluate the behavioral profile of the mortality rate (TM) due to prostate cancer (CP) in the Northeast region, considering the period from 1988 to 2017, as well as the progression of the TM to the period from 2018 to 2067. METHODOLOGY: For this, a documentary and descriptive study was conducted, based on data collected in the Atlas of Mortality on TM for prostate cancer of the northeastern population from 1988 to 2017, and from these data was calculated the progression of mortality by this disease. Results for the period 2018 to 2067. RESULTS: During the period analyzed (1988 to 2017), it was observed that mortality due to CP increased continuously, making the Northeast the second region of Brazil with the highest MT by CP in the last decade of studies ( Estimating the progression of mortality due to $P C$ over the 50-year period, it was found that it tends to increase from 33.17 to 689.28 in the period from 2018 to 2067, respectively. than that presented in the progression of breast cancer TM in the 90-year period in that region. CONCLUSION: Thus, it is concluded that it is necessary to implement measures and actions related to men's health, aiming to regress the TM by PC. It becomes apparent that otherwise this rate tends to rise sharply in subsequent years.

Keywords: Prostate Cancer; Prevention; Men's Health. 


\section{INTRODUÇÃO}

No Brasil, o câncer de próstata é o segundo mais comum entre os homens (atrás apenas do câncer de pele não-melanoma). Em valores absolutos e considerando ambos os sexos, é o segundo tipo mais comum. A taxa de incidência é maior nos países desenvolvidos em comparação aos países em desenvolvimento. Mais do que qualquer outro tipo, é considerado um câncer da terceira idade, já que cerca de $75 \%$ dos casos no mundo ocorrem a partir dos 65 anos. O aumento observado nas taxas de incidência no Brasil pode ser parcialmente justificado pela evolução dos métodos diagnósticos (exames), pela melhoria na qualidade dos sistemas de informação do país e pelo aumento na expectativa de vida. (INCA, 2019).

A próstata é uma glândula que só o homem possui e que se localiza na parte baixa do abdômen. Ela é um órgão pequeno, tem a forma de maçã e se situa logo abaixo da bexiga e à frente do reto (parte final do intestino grosso). A próstata envolve a porção inicial da uretra, tubo pelo qual a urina armazenada na bexiga é eliminada. (INCA, 2019).

Sabe-se que comumente a história natural desta doença é lenta, sendo possível conviver com o tumor por um considerável espaço de tempo, ainda se faz necessário que as devidas ações preventivas visando a identificação precoce sejam realizadas periodicamente, pelo fato de existirem tumores que se desenvolvem de forma muito agressiva e possuem facilidade para se disseminar, o que aumenta consideravelmente o risco de morte do indivíduo afetado.

A promoção de saúde para o público masculino é de fundamental importância, pois ainda se observa que os homens possuem déficit de autocuidado, associado a pensamentos ultrapassados e preconceituosos, dificultando a adesão aos métodos de prevenção e favorecendo o estabelecimento de patologias, como o câncer de próstata (CP) que é uma das principais causas de morte na população masculina. 
Assim, ao analisar o comportamento masculino percebe-se claramente obstáculos que distanciam os homens da prevenção, tais como: a cultura em que estão inseridos e até alguns fundamentos machistas ainda enraizados, a falta de conhecimento esclarecedor quanto aos exames, e também a carência de mais ações informativas que mostrem ao público a importância de submeter-se a exames preventivos de rotina, esclarecendo também todas as dúvidas que sejam impostas, formando assim uma relação de confiança entre profissional de saúde e paciente, favorecendo assim que as informações passadas não sejam ignoradas, e resultem em melhores prognósticos para os pacientes, diminuindo desse modo a taxa de mortalidade. Desta forma, o objetivo deste estudo foi avaliar o perfil de comportamento da taxa de mortalidade por CP na região Nordeste, considerando o período de 1988 à 2017, assim como a progressão da taxa de mortalidade para o período 2018 à 2067, avaliando-se posteriormente a progressão dessa mortalidade.

\section{MATERIAIS E MÉTODO}

Este trata-se de um estudo documental-descritivo, e para desenvolve-lo foi utilizado como base dados colhidos no Atlas de Mortalidade On-line do INCA em julho de 2019, referentes a taxa de mortalidade por câncer de próstata durante o período de três décadas, sendo elas: 1988-1997; 1998-2007; 2008-2017, estes dados foram analisados, identificando-se desse modo os aumentos entre as décadas e elaborando-se posteriormente uma progressão ao longo de cinquenta anos, que foi calculada a partir das taxas de progressão observada nos dados previamente colocados, referentes aos últimos 30 anos.

\section{RESULTADOS E DISCUSSÃO}


De acordo com a (figura I), verifica-se a gradativa evolução da TM por CP na região Nordeste durante os períodos analisados, onde conclui-se que essa região mudou de posição ao longo das décadas, em relação a TM por essa neoplasia, iniciando a primeira década (1988-1997) com taxa de 3,41/100 mil homens ,situando-se em $5^{\circ}$ lugar em comparação as demais regiões do Brasil, na segunda década (1998-2007) com taxa de 7,69/100 mil homens, encontrando-se em 4 \%lugar e encerrou a terceira década analisada (2008-2017) com taxa de 15,54/100 mil homens, passando a ocupar o $2^{\circ}$ lugar, frente a isso pode-se constatar que os aumentos sofridos nessa região foram extremamente consideráveis. Levando-se em consideração esses aspectos, é possível dizer que o Nordeste se tornou a segunda região do Brasil com maior taxa de mortalidade por câncer de próstata na última década estudada (2008-2017).

Figura I: Taxas de mortalidade por câncer de próstata, para cada 100 mil homens, na região Nordeste.

\begin{tabular}{c|c|c|c|}
\hline Região & $1988-1997$ & $1998-2007$ & $2008-2017$ \\
\hline Norte & 3,76 & 6,84 & 14,01 \\
\hline Nordeste & 3,41 & 7,69 & 15,54 \\
\hline Centro-oeste & 6,77 & 11,30 & 15,79 \\
\hline Sul & 8,50 & 13,05 & 15,20 \\
\hline Sudeste & 8,05 & 11,50 & 13,26 \\
\hline
\end{tabular}

Fonte: O autor, 2019.

A Política Nacional de Atenção Integral à Saúde do Homem traduz um longo anseio da sociedade ao reconhecer que os agravos do sexo masculino constituem verdadeiros problemas de saúde pública. Um dos principais objetivos desta Política é promover ações de saúde que contribuam significativamente para a compreensão da realidade singular masculina nos seus diversos contextos socioculturais e político-econômicos; outro, é o respeito aos diferentes níveis de desenvolvimento e organização dos sistemas locais de saúde e tipos de gestão. Este conjunto possibilita o aumento da expectativa de vida e a redução dos índices de morbimortalidade por causas preveníveis e evitáveis nessa população. (MINISTÉRIO DA SAÚDE, 2008). Criada em 2009, essa política procura incluir a masculinidade nas questões clínica e epidemiológica, oferecendo uma proposta 
singular de cuidado de promoção e recuperação da saúde. (TRILICO, 2015.). De acordo com a (figura II), observa-se um aumento na TM por CP no Nordeste, sendo observado uma taxa de mortalidade de 3,41 para cada 100 mil homens, no período de 1988 à 1997, e uma taxa de 15,54 para cada 100 mil homens, no período de 2008 à 2017, o que seria justificável no período de 1988 à 2009, onde ainda não se tratava a população masculina de forma separada, atentando-se para os indicadores epidemiológicos que refletem a qualidade de saúde dessa população, entretanto, é notório que embora desde 2009 à saúde do homem tenha começado a ser vista de forma mais específica, a mortalidade por CP na região segue elevando-se até o final do período analisado, 2017.

Figura II. Mortalidade masculina por câncer de próstata para cada 100 mil homens, na região Nordeste, por taxa ajustada, no período de trinta anos, 1988-1997; 1998$2007 ; 2008-2017$.

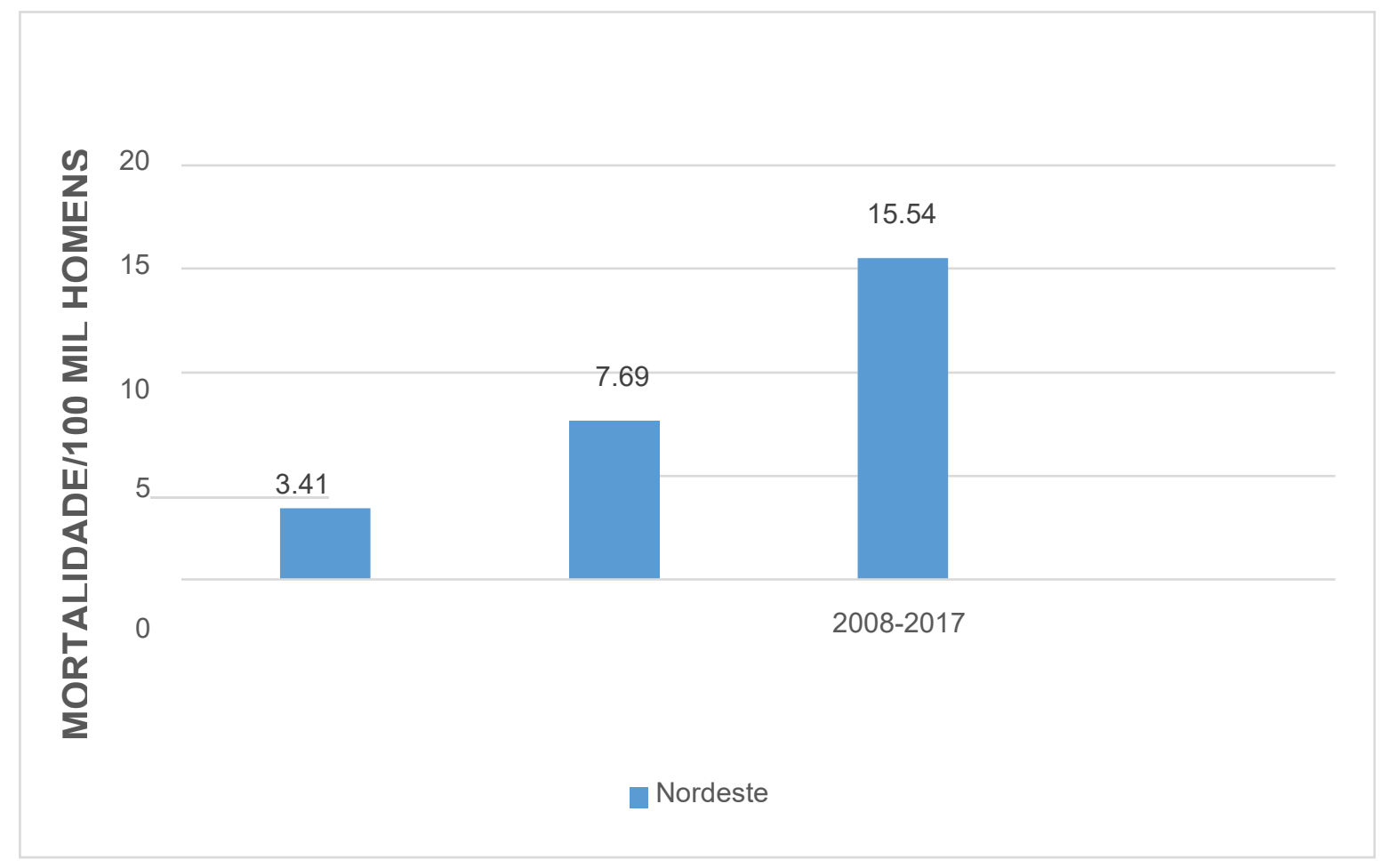

Fonte: O autor, 2019. 
Diante do exposto, sugere-se que existem dois fatores principais que são influenciadores diretos na baixa adesão do público masculino as práticas preventivas, e consequentemente no aumento progressivo da mortalidade por $\mathrm{CP}$ durante o período analisado (1988-2017), sendo os prováveis fatores influenciadores: déficit de autocuidado e um possível serviço de saúde deficitário. Tradicionalmente, os homens não têm suas especificidades reconhecidas e não fazem parte das populações usualmente mais assistidas nos serviços de atenção básica à saúde (ABS). O uso dos serviços de saúde pelos homens difere daquele feito pelas mulheres, concentrando-se na assistência a agravos e doenças, em que a busca por atendimento, em geral, acontece em situações extremas de emergência e/ou em nível especializado ou de urgência (CASTRO; TOCANTINS, 2010). Portanto, ao reportar-se a saúde do homem, evidencia-se que parte dos homens mantém preferência por não procurar frequentemente serviços de saúde, principalmente tratando-se da busca por serviços preventivos, favorecendo assim consequências como o aumento da TM por câncer de próstata, tal consequência é reflexo de um certo déficit de autocuidado ainda presente em diversos homens.

Uma questão apontada pelos homens para a não procura pelos serviços de saúde está ligada à sua posição de provedor. Alegam que o horário do funcionamento dos serviços coincide com a carga horária do trabalho. Não se pode negar que na preocupação masculina a atividade laboral tem um lugar destacado, sobretudo em pessoas de baixa condição social o que reforça o papel historicamente atribuído ao homem de ser responsável pelo sustento da família. Ainda que isso possa se constituir, em muitos casos, uma barreira importante, há de se destacar que grande parte das mulheres, de todas as categorias socioeconômicas, faz hoje parte da força produtiva, inseridas no mercado de trabalho, e nem por isso deixam de procurar os serviços de saúde (MINISTÉRIO DA SAÚDE, 2008). Além disso, os serviços e as estratégias de comunicação privilegiam as ações de saúde para a criança, o adolescente, a mulher e o idoso (MINISTÉRIO DA SAÚDE, 2008). Fazendo que desse modo, o público masculino se torna cada vez mais distante das estratégias preventivas, pois embora tenham conhecimento da importância de realizar as práticas de prevenção, não são abordados de forma adequada, o que gera uma possível barreira entre o serviço de saúde e o usuário. 
A atenção à saúde do homem foi por muito tempo negligenciada pelos diferentes setores da saúde, dos diversos níveis governamentais. Entretanto, contemporaneamente, com a aprovação da Política Nacional de Assistência à Saúde do Homem, verificou -se a ocorrência crescente de discussões que envolvem o processo saúde-doença da clientela masculina (MOREIRA, et al., 2014).

Grande parte da população masculina se encontra em estado de inércia quando se trata de autocuidado, visto que poucos procuram a unidade de saúde, e geralmente os mesmos tendem a procurá-las apenas quando apresentam dores insuportáveis, que os deixa mais vulneráveis, propiciando a evolução da doença para estágios munidos de complicações de difícil tratamento, podendo gerar casos irreversíveis. Alguns homens têm dificuldade em reconhecer e aceitar suas fragilidades, por isso buscam os serviços de saúde esporadicamente. Isto pode estar associado em questão a sociedade brasileira ser estruturada pela ideologia do patriarcado que legitima a "superioridade" do homem, influenciando-os direta ou indiretamente pelas ideias hegemônicas. Por esse fato, cuidam pouco da saúde e demoram a procurar o médico, haja vista que o cuidado com a saúde pode ser considerado sinal de fracasso (PEREIRA, et al., 2015).

Verifica-se, pois, que os homens acessam os serviços de saúde por meio da atenção terciária, quando já existe um quadro clínico de morbidade instalado, muitas vezes cronificado. Demandando altos custos sociais, particularmente, para os homens, e econômicos para o estado (MOREIRA, et al.,2014). Frente a isso, o aperfeiçoamento nos serviços preventivos para manter a saúde do homem, além de ser imprescindível para diminuir a crescente taxa de mortalidade por $\mathrm{CP}$, sendo também importante para reduzir gastos do estado com as possíveis complicações da doença descoberta tardiamente.

Utilizando-se da média de aumento entre as décadas, foi estimada a progressão da taxa de mortalidade por CP em um período de 50 anos (2018-2067), possibilitando assim observar qual seria o comportamento da mortalidade por $\mathrm{CP}$ caso não haja melhorias e eficácia nas intervenções utilizadas atualmente para prevenir a neoplasia.

De acordo com a (Figura III), percebe-se que na última década, 2058 à 2067, do período de 50 anos, teríamos uma taxa de mortalidade de 689,28 mortes para 
cada 100 mil homens (Figura 3), o que será previamente esperado, caso a adesão da população masculina aos exames não se tornem mais frequentes nesse período, pois nos dias atuais, fica claro que as ações de saúde não estão sendo tão eficazes quando deveriam se tratando de mobilizar o público masculino sobre a importância de realizar exames preventivos periodicamente, principalmente idosos da terceira idade, que se enquadram na população com maior mortalidade pela neoplasia (INCA, 2019).

Pelo fato de as neoplasias malignas prostáticas serem geralmente silenciosas no início, levando os indivíduos a não procurarem assistência médica, por associarem o fato de ausência de sintomas com ausência de doença, sendo assim, a patologia é identificada em estágio inicial principalmente em indivíduos que possuem a prática de realizar exames de rotina, a exemplo da dosagem do antígeno prostática específico (PSA), elevando assim as chances de cura, porém, os prognósticos dos pacientes que a identificam tardiamente tende a ser desfavorável, desse modo percebe-se a importância de buscar estratégias preventivas eficazes e de fácil a aplicabilidade para reverter o número de mortes registradas pela doença. 
Figura III: Progressão da taxa de mortalidade por câncer de próstata para cada 100 mil homens na região Nordeste, no período de 50 anos (2018 à 2067).

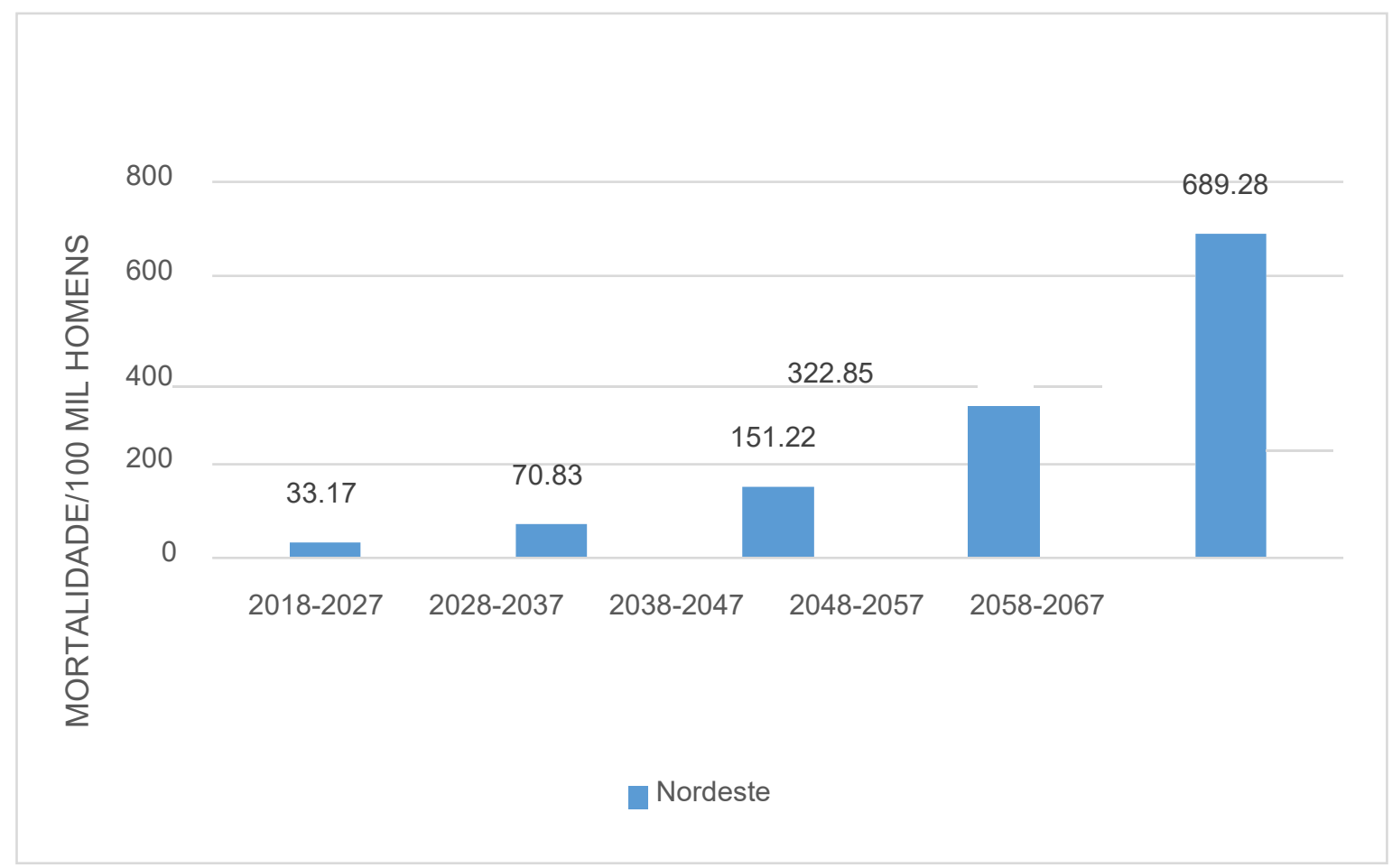

Fonte: O autor, 2019.

Nota-se que a tendência da mortalidade por CP na região Nordeste seria aumentar a cada década subsequente, caso a situação das intervenções preventivas realizadas na região permaneçam com padrões insuficientes. Fazendose uma comparação entre a progressão do CP e a progressão do Câncer de Mama (CM) na região Nordeste, é notório que a projeção da taxa de mortalidade por CP em 50 anos seria 689,28/100 mil homens, sendo esse valor superior a taxa de mortalidade por CM em 90 anos (2017-2107), que seria 135,16 /100 mil mulheres, o que nos leva a afirmar que a prevenção do CM está sendo mais eficaz quando comparada a prevenção do $\mathrm{CP}$, considerando-se que tanto o gênero feminino quanto o masculino encontram-se nas mesmas condições de exposição e tratamento (MACÊDO; TERCEIRO; DANTAS, 2019).

Os serviços de saúde também são considerados pouco aptos em absorver a demanda apresentada pelos homens, pois sua organização não estimula o acesso e 
as próprias campanhas de saúde pública não se voltam para este segmento. Além disso, o mercado de trabalho geralmente não garante formalmente a adoção de tal prática, portanto, o homem em dado momento pode ser prejudicado e sentir seu papel de provedor ameaçado. Outra dificuldade para o acesso dos homens a esses serviços é a vergonha da exposição do seu corpo perante o profissional de saúde, particularmente a região anal, no caso da prevenção ao câncer de próstata. Também é apontado como um fator que dificulta esse acesso a falta de unidades específicas para o tratamento da saúde do homem. (GOMES, 2007). Estes fatores contribuem diretamente para o afastamento do público masculino em relação às unidades de saúde, privando-os de um possível diagnostico inicial do CP, caracterizando identificações tardias da doença, com maior possibilidade de mal prognóstico, culminando em descontrole e possivelmente aumentos da taxa de mortalidade por essa patologia.

Segundo o Ministério da Saúde, estudos sobre a saúde dos homens vêm ganhando destaque no cenário nacional devido às elevadas taxas de mortalidade e morbidade que afetam esse grupo, assim como a sua baixa procura pelos serviços de atenção primária à saúde (MENDONÇA; ANDRADE, 2010). Desse modo, por ainda haver uma baixa procura pelos serviços de saúde, gerando consequentemente um menor número de homens que procuram realizar exames de prevenção, caracterizando identificações tardias da doença, com maior possibilidade de mal prognóstico, culminando em descontrole e possivelmente aumentos da taxa de mortalidade por essa patologia.

A prevenção primária, a ser desenvolvida no período de pré-patogênese, consta de medidas destinadas a desenvolver uma saúde geral melhor, pela proteção específica do homem contra agentes patológicos ou pelo estabelecimento de barreiras contra os agentes do meio ambiente. A educação em saúde é elemento importante para esse objetivo. Afirmam os autores que os procedimentos para a promoção da saúde incluem um bom padrão de nutrição, ajustado às várias fases do desenvolvimento humano; o atendimento das necessidades para o desenvolvimento ótimo da personalidade, incluindo o aconselhamento e educação adequados dos pais, em atividades individuais ou de grupos; educação sexual e aconselhamento pré-nupcial; moradia adequada; recreação e condições agradáveis no lar e no 
trabalho. A orientação sanitária nos exames de saúde periódicos e o aconselhamento para a saúde em qualquer oportunidade de contato entre o médico e o paciente, com extensão ao resto da família, estão entre os componentes da promoção (BUSS, 2000). Desse modo, é necessário fortalecer e qualificar a atenção primária garantindo, assim, a promoção da saúde e a prevenção aos agravos evitáveis. Muitos agravos poderiam ser evitados caso os homens realizassem, com regularidade, as medidas de prevenção primária. À resistência masculina a atenção primária aumenta não somente a sobrecarga financeira da sociedade, mas também, sobretudo, o sofrimento físico e emocional do paciente e de sua família, na luta pela conservação da saúde e da qualidade de vida dessas pessoas (MINISTERIO DA SAÚDE, 2008).

\section{CONCLUSÕES}

Em face aos aspectos analisados, pode-se dizer que a prevenção do câncer de próstata não está sendo realizada de forma eficaz, visto que a taxa de mortalidade pela a neoplasia em questão continuou progredindo durante todo o período analisado, considerando-se também que ao analisar a progressão de 50 anos a taxa de mortalidade por essa neoplasia pode chegar a valores extremos, portanto, se faz necessário que as intervenções de saúde tenham um maior foco no público masculino, visto que o mesmo se mostra mais vulnerável a ir a óbito por não procurar os serviços de saúde em busca de exames preventivos, a fim de evitar o aparecimento de patologias ou identificar precocemente as mesmas, para que dessa forma as possibilidades de cura sejam ampliadas, regredindo desse modo a taxa de mortalidade por câncer de próstata , o que refletiria na estimativa para a taxa de mortalidade nos anos seguintes. 


\section{REFERÊNCIAS BIBLIOGRÁFICAS}

AGUIAR, Milena Cabral de; ALMEIDA, Obertal da Silva. A implantação da política nacional de atenção integral à saúde do homem no Brasil: um desafio para a saúde pública. Revista Diálogos \& Ciência n 30, junho de 2012. Disponível em: $<$ https://www.researchgate.net/publication/272883065_A_implantacao_da_politica_na cional_de_atencao_integral_a_saude_do_homem_no_Brasil_um_desafio_para_a_saude_p ublica>. Acesso em: 20 Dez. 2019.

ALVES, Ranilda Fernandes et al. Gênero e saúde: o cuidar do homem em debate. Revista Psicologia: Teoria e Prática - 2011.2 Disponível em: <https://www.redalyc.org/articulo.oa?id=193821358012>. Acesso em: 20 Dez. 2019.

BRASIL. Ministério da Saúde, Secretaria de atenção à saúde, Departamento de ações programáticas estratégicas. Política nacional de atenção integral à saúde do homem. Brasília, novembro de 2008.2 Disponível em:<https://www.google.com/url?sa=t\&source=web\&rct=j\&url=http://bvsms.saude.gov.br/bvs/pub licacoes/politica_nacional_atencao_saude_homem.pdf\&ved=2ahUKEwiY_q- ctu3mAhVFLkGHY9mCAYQFjAAegQIBhAB\&usg=AŌVVaw2IHAYkpjYKISSTwbz_Oh43>. Acesso em: 20 Dez. 2019.

BUSS, Paulo Marchiori. Promoção da saúde e qualidade de vida. Ciência \& saúde coletiva vol.5 no.1, P.166, Rio de Janeiro 2000. Disponível em:<http://dx.doi.org/10.1590/S141381232000000100014>. Acesso em: 22 Nov. 2019.

CASTRO, Caroline Oliveira de; TOCANTINS, Florence Romijn. Necessidades assistenciais do homem na perspectiva da Enfermagem e a saúde da família. Revista pesquisa.: cuidado é fundamental online 2010. out/dez. 2(Ed. Supl.):813-816. Disponível em: $<$ http://www.seer.unirio.br/index.php/cuidadofundamental/article/viewArticle/1141>. Acesso em: 20 nov. 2019.

INSTITUTO NACIONAL DO CANCER (INCA/MS) PRÓ-ONCO. Informativo Detecção Precoce. Boletim ano 8, $n^{\circ} \quad 2$, p.1 julho/dezembro 2017. Disponível em: $<$ https://www.inca.gov.br/publicacoes/informativos/informativo-deteccao-precoce-no- 2-2017>. Acesso em: 21 Nov. 2019.

FIGUEIREDO, Wagner. Assistência à saúde dos homens: um desafio para os serviços de atenção primária. Revista Ciência \& Saúde Coletiva, Rio de Janeiro, Ago. 2004. Disponível em: <https://www.scielosp.org/article/csc/2005.v10n1/105-109/es/>. Acesso em: 02 Jan. 2020.

GARÓFOLO, Adriana et al. Dieta e câncer: um enfoque epidemiológico. Revista de Nutrição vol.17 no.4 Campinas Oct/Dec. 2004. Disponível em:< http://dx.doi.org/10.1590/S141552732004000400009>. Acesso em: 23 Dez. 2019.

GOMES, Romeu et al. A prevenção do câncer de próstata: uma revisão da literatura. Revista Ciência \& Saúde Coletiva vol.13 no.1 Rio de Janeiro, p.236, Jan./Feb. 2008. Disponível em: < http://dx.doi.org/10.1590/S1413-81232008000100027>. Acesso em: 21 Nov. 2019.

GOMES, Romeu et al. Por que os homens buscam menos os serviços de saúde do que as mulheres? As explicações de homens com baixa escolaridade e homens com ensino superior. Cadernos de Saúde Pública[online]. 2007, vol.23, n.3, p.565. ISSN 0102-311X. Disponível em: <http://dx.doi.org/10.1590/S0102-311X2007000300015>. Acesso em: 28 Nov. 2019. 
GONCALVES, Ivana Regina; PADOVANI, Carlos and POPIM, Regina Célia. Caracterização epidemiológica e demográfica de homens com câncer de próstata. Ciênc. saúde coletiva [online]. 2008, vol.13, n.4, pp.1337-1342. ISSN 1413- 8123. http://dx.doi.org/10.1590/S141381232008000400031.

JÚNIOR, Arilton Januário Bacelar et al. Câncer de próstata: métodos de diagnóstico, prevenção e tratamento. Brazilian Journal of Surgery and Clinical Research - BJSCR. Vol.10, n.3, pp. 40-46, Mar - Mai 2015. Disponível em: < https://www.mastereditora.com.br/periodico/20150501_174533.pdf> Acesso em: 29 Dez. 2019.

LAURENTI, Ruy et al. Perfil epidemiológico da morbi-mortalidade masculina. Ciência \& Saúde Coletiva, Rio de Janeiro, 2005. Disponível em: $<$ https://scholar.google.com.br/scholar?hl=pt-

PT\&as_sdt $=0 \% 2 \mathrm{C} 5 \& \mathrm{q}=$ Constructions+of + masculinity+and+their+influence + on + men $\% E 2 \% 80 \% 9$ $9 s+$ well-+being $\% 3 \mathrm{~A}+\mathrm{a}+$ theory+of+gender+and+health\&btnG=\#d=gs_qabs\&u=\%23p\%3DyHEvK W9zLLwJ>. Acesso em: 29 Dez. 2019.

LEITE, Denise Fernandes et al. A influência de um programa de educação na saúde do homem. Revista O Mundo da Saúde, São Paulo, p.51, 2010. Disponível em: $<$ https://www.google.com/url?sa=t\&source=web\&rct=j\&url=http://www.saocamilo-

sp.br/pdf/mundo_saude/74/06_original_influencia.pdf\&ved=2ahUKEwjZuMfKte3mAhW-

EbkGHZveBrIQFjAAegQIAhABB\&usg=AOvVawOIVm7IV7QWccVh401IzyxK>. Acesso em: 01 Jan. 2020.

MACÊDO, Jéssyka Samara de Oliveira; TERCEIRO, Lucas Evangelista de Lima; DANTAS, Bruna Braga. Câncer de mama: análise da mortalidade e perspectiva de tratamento. Revista de Ciências da Saúde Nova Esperança Volume 17, número 2, p.12, Ago de 2019. Disponível em: <https://doi.org/10.17695/revnevol17n2p06-18>. Acesso em: 29 Dez. 2019.

MACHIN, Rosana et al. Concepções de gênero, masculinidade e cuidados em saúde: estudo com profissionais de saúde da atenção primária. Revista Ciência \& Saúde Coletiva, p.4505, Rio de Janeiro, jan de 2010. Disponível em: $<$ https://scholar.google.com.br/scholar?hl=pt-

PT\&as_sdt $=0 \% 2 \mathrm{C} 5 \& \mathrm{q}=$ Concep $\% \mathrm{C} 3 \% \mathrm{~A} 7 \% \mathrm{C} 3 \% \mathrm{~A} 3 \mathrm{o}+\mathrm{de}+\mathrm{g} \% \mathrm{C} 3 \% \mathrm{AAnero}+\% 2 \mathrm{Cmasculinidade}+\mathrm{e}$ +cuidados+em+sa\%C3\%BAde $\% 3 \mathrm{~A}+$ estudo+com+profissionais+de+sa\% $3 \%$ BAde+da+aten $\% \mathrm{C}$ $3 \% \mathrm{~A} 7 \% \mathrm{C} 3 \% \mathrm{~A} 30+$ prim\%C3\%A1ria\&btnG=\#d=gs_qabs\&u=\%23p\%3DqM NIAuSuPngJ>. Acesso em: 21 Dez. 2019.

MENDONÇA, Vítor Silva; ANDRADE, Angela Nobre de. A Política Nacional de Saúde do Homem: necessidade ou ilusão? Revista psicológica política. vol.10 no.20, p.217, São Paulo dez. 2010. Disponível em:< http://pepsic.bvsalud.org/scielo.php?script=sci_arttext\&pid=S1519549X2010000200003>. Acesso em: 01 Dez. 2019.

MINISTÉRIO DA SAÚDE. Atlas de Mortalidade, 1996-2014. INCA. Disponivel em:<https://mortalidade.inca.gov.br/MortalidadeWeb/>. Acesso em: Julho de 2019.

MOURA, Erly Catarina de et al. Atenção à saúde dos homens no âmbito da Estratégia Saúde da Família. Ciência \& Saúde coletiva vol.19 no.2, p.430, Rio de Janeiro Feb. 2014. Disponível em:< http://dx.doi.org/10.1590/1413-81232014192.05802013>. Acesso em: 27 Dez. 2019.

MOREIRA, Renata Lívia Silva Fonsêca; FONTES, Wilma Dias de e BARBOZA, Talita Maia. Dificuldades de inserção do homem na atenção básica a saúde: a fala dos enfermeiros. Esc. Anna Nery [online]. 2014, vol.18, n.4, p.616. ISSN 1414- 8145. Disponível em: <http://dx.doi.org/10.5935/1414-8145.20140087>. Acesso em: 29 Out. 2019.

PEREIRA, Marcia Maria Marques et al. Saúde do homem na atenção básica: análise acerca 
do perfil e agravos à saúde. Revista de enfermagem, UFPE online., p.441, Recife, 9(supl.1):440-7. jan., 2015. Disponível em: <https://www.google.com/url?sa=t\&source=web\&rct=j\&url=https://periodicos.ufpe.br/revi stas/revistaenfermagem/article/download/10357/11079\&ved=2ahUKEwjeu5Xjtu3mAhVk HLkGHUMGCTAQFjAAegQIBhAB\&usg=AOvVaw1BOeJzFQI6DhTC72iVIvXn>. Acesso em: 27 Out. 2019.

PINHEIRO, Rejane Sobrino et al. Gênero, morbidade, acesso e utilização de serviços de saúde no Brasil. Ciência \& Saúde Coletiva. 2002. Disponível em: <https://www.scielosp.org/article/csc/2002.v7n4/687-707/>. Acesso em: 28 Dez. 2019.

SILVA, Patrick Leonardo Nogueira da et al. A Política de Atenção à Saúde do Homem no Brasil e os desafios da sua implantação: uma revisão integrativa. Revista Eletrônica Trimestral de Enferméria $\mathrm{N}^{\circ}$ 32, p.415, October 2013. Disponível em: $<$ https://www.google.com/url?sa=t\&source=web\&rct=j\&url=http://scielo.isciii.es/pdf/eg/v 12n32/pt_enfermeria3.pdf\&ved=2ahUKEwipo9qAtu3mAhUJI7kGHZOWDdgQFjAAegQ IAxAB\&usg=AOvVaw2rE7lyJwY-s8lcUgOXjWqP>. Acesso em: 22 Nov. 2019.

SOUZA, Karolayne Silva et al. Tendência de Mortalidade por câncer de próstata na região Nordeste do Brasil, 1996 - 2014. Revista Rios Saúde, p.19-20, 2018. Disponível em: < https://www.unirios.edu.br/revistariossaude/>. Acesso em: 27 Dez. 2019.

TRILICO, Matheus Luis Castelan et al. Discursos masculinos sobre prevenção e promoção da saúde do homem. Revista Trabalho, Educação e Saúde, Rio de Janeiro, v. 13 n. 2, p. 382, maio/ago. 2015. Disponível em: <http://dx.doi.org/10.1590/1981-7746-sip00015>. Acesso em: 30 Dez. 2019. 